\title{
Impact test comparisons of 20th and 21st century American football helmets
}

\author{
Laboratory investigation
}

\author{
Adam Bartsch, Ph.D., ${ }^{1,2}$ Edward Benzel, M.D. ${ }^{1-3}$ Vincent Miele, M.D., $2-4$ \\ and Vikas Prakash, Ph.D. ${ }^{2,5}$ \\ ${ }^{I}$ Spine Research Laboratory; ${ }^{3}$ Department of Neurosurgery, Neurological Institute, Cleveland Clinic; \\ ${ }^{2}$ Cleveland Traumatic Neuromechanics Consortium; ${ }^{5}$ Department of Mechanical Engineering, Case \\ Western Reserve University, Cleveland, Ohio; and ${ }^{4}$ United Hospital Center Neuro-Spine Center, \\ Bridgeport, West Virginia
}

\begin{abstract}
Object. Concussion is the signature American football injury of the 21 st century. Modern varsity helmets, as compared with vintage leather helmets, or "leatherheads," are widely believed to universally improve protection by reducing head impact doses and head injury risk for the 3 million young football players in the US. The object of this study was to compare the head impact doses and injury risks with 11 widely used 21 st century varsity helmets and 2 early 20th century leatherheads and to hypothesize what the results might mean for children wearing similar varsity helmets.

Methods. In an injury biomechanics laboratory, the authors conducted front, oblique front, lateral, oblique rear, and rear head impact tests at $5.0 \mathrm{~m} /$ second using helmeted headforms, inducing near- and subconcussive head impact doses on par with approximately the 95th percentile of on-field collision severity. They also calculated impact dose injury risk parameters common to laboratory and on-field traumatic neuromechanics: linear acceleration, angular acceleration, angular velocity, Gadd Severity Index, diffuse axonal injury, acute subdural hematoma, and brain contusion.

Results. In many instances the head impact doses and head injury risks while wearing vintage leatherheads were comparable to or better than those while wearing several widely used 21 st century varsity helmets.

Conclusions. The authors do not advocate reverting to leather headgear, but they do strongly recommend, especially for young players, instituting helmet safety designs and testing standards, which encourage the minimization of linear and angular impact doses and injury risks in near- and subconcussive head impacts.
\end{abstract}

(DOI: 10.3171/2011.9.JNS111059)

KEY WORDS $\bullet$ concussion $\bullet$ American football $\bullet$ football helmet
high school football $\bullet \quad$ youth football $\bullet \quad$ traumatic brain injury

A VAILABLE data and statistics regarding concussion and TBI vary widely. Nevertheless, it has been estimated that approximately 300,000 sports-related concussions $\mathrm{s}^{53}$ and upwards of 3.8 million sport- and recreation-related $\mathrm{TBIs}^{28}$ occur annually in the US, with direct medical and indirect costs in the tens of billions yearly. ${ }^{16}$ American football is the leading cause of sportsrelated concussion in the US. ${ }^{19}$ And the concussion crisis is underestimated, as evidenced by the fact that approxi-

\footnotetext{
Abbreviations used in this paper: AIS = Abbreviated Injury Scale; CSDM05 = cumulative strain damage measure; DAI = diffuse axonal injury; DDM = dilatational damage measure; GSI = Gadd Severity Index; NFL = National Football League; NOCSAE $=$ National Operating Committee on Standards for Athletic Equipment; RMDM $=$ relative motion damage measure; SIMon $=$ Simulated Injury Monitor; TBI = traumatic brain injury.
}

mately 53\% of American football-related concussions in high school go unreported. ${ }^{30}$

The term "concussion" has been used to describe a gamut of injuries and disorders resulting from the traditional acute symptomatic concussion from a single head impact, including rare cases of transient loss of consciousness (reported in $<1$ in 10 concussed athletes ${ }^{23,48}$ ) and more frequent neurological dysfunctions such as headache, dizziness, confusion, disorientation, blurred vision, balance deficits, delayed reaction time, amnesia, sleep disturbances, neck pain, and fatigue. ${ }^{31}$ Further, clinically silent subconcussive injuries from accumulated head impact doses over months, years, or decades can be present. Both types of injuries can cause reversible as well as irreversible brain damage. Although concussion has been widely described as a type of TBI, mild TBI, concussive TBI, mild head injury, closed head injury, minor head trauma, subconcussive 
impact, ding, bell-ringer, or knockout, in the present study we defined concussion as incorporating all of the aforementioned terms and descriptions. Moreover, according to this injury severity definition, head injury severity must be consistent with a clinically diagnosed AIS ${ }^{18}$ score of 1 (mild cerebral concussion without loss of consciousness) or 2 (cerebral concussion with loss of consciousness $<30$ minutes) or with clinically silent subconcussive injuries.

There is increasingly intense government and media focus on the brain health implications of concussion in youth, collegiate, and professional American football players. Recently, as a possible sequela of the cumulative effect of clinically symptomatic concussive and clinically silent subconcussive impacts, the careers, and in some cases the lives, of a number of high-profile football players have ended prematurely. Concerns regarding the immediate and long-term effects of multiple concussive and subconcussive impacts on the brain health of football players have led to escalating demands for improvements in helmet design and testing standards, ${ }^{7,8}$ as well as for a reevaluation of athlete training regimens and on-field safety-related rules and regulations. ., $^{4,10,49,56}$ Despite this heightened awareness surrounding concussion, much remains unknown about the risks of football-related head impact doses. Furthermore, only recently have American football leagues begun instituting safety measures to restrict head impact doses by limiting the number of impacts sustained in practices or games (http://www. nytimes.com/2011/07/20/sports/ncaafootball/collegefootball-to-protect-players-ivy-league-to-reduce-contact. $\mathrm{html}$ ). (Note that youth baseball has for years provided a model for injury risk reduction via discrete pitch counts to limit upper-extremity overuse.) Most importantly, in 2011, the US Congress recognized the urgent need to help prevent potential concussion in millions of children playing tackle football by introducing legislation to mandate standard means of evaluating youth football helmet protectiveness. ${ }^{7,8}$

While the NOCSAE standard has done much to advance 20th century football helmet protectiveness in higher-dose impacts responsible for catastrophic injury (helmet-to-anvil impacts resulting in several hundred times the force of gravity, or $\mathrm{g}$ units), the more frequent on-field impacts occurring at a lower dose (helmet-tohelmet impacts $<75 \mathrm{~g}$ ) have yet to be studied in depth in a controlled laboratory setting. Furthermore, since youth helmets are scaled-down versions of varsity helmets for adults, the lack of knowledge surrounding adult helmet protection against lower-energy dose impacts, as well as the absence of any youth-specific helmet testing standards, may have serious brain health implications for the 3 million youths playing tackle football in the US annually (http://usafootball.com/sites/default/files/USA_Football_ one_pager_Dec_2010.pdf). To address the clinical significance of common lower-dose on-field impacts (for example, near and subconcussive head impacts), we critically analyzed in a controlled laboratory setting the protectiveness of all 11 available 21st century varsity helmets meeting the NOCSAE standard as well as 2 vintage leather helmets. This analysis was also performed to gain further insight into helmet designs and testing standards that minimize impact dose and injury risk for players of all ages. Therefore, rather than focusing solely on linear acceleration like the current NOCSAE standard, we focused on a multitude of important laboratory and on-field traumatic neuromechanics injury risk parameters: linear acceleration, angular acceleration, angular velocity, GSI, DAI, acute subdural hematoma, and brain contusion.

\section{Methods}

\section{Our Study}

In our testing, a stationary helmeted NOCSAE headform (size medium, Southern Impact Research Center, LLC) was attached to a Hybrid III male neckform (50th percentile, Humanetics Innovative Solutions) and was struck by a swinging NOCSAE headform (size medium) fitted with a Riddell VSR-4 varsity helmet (adult size large). The total pendulum swing weight was 6.2 $\mathrm{kg}$. Eleven varsity helmets with polycarbonate shell and skill position face masks and meeting the NOCSAE football helmet standards ${ }^{38,39}$ (Table 1) were obtained from 4 manufacturers, were fitted on the stationary headform, and were struck by the swinging helmeted headform. Additionally, 2 vintage 20 th century leather football helmets without face masks were fitted on the stationary headform and tested.

The NOCSAE headform was instrumented with 3 uniaxial 500g linear accelerometers (model M62, Measurement Specialties) in an orthogonal arrangement at the headform's center of gravity and with 3 uniaxial 210-radians/second angular velocity sensors (Diversified Technical Systems). For the angular velocity sensors, a planar rotation transform was implemented to transform the measured angular velocity into the linear accelerometer local reference frame. The base of the neck was bolted directly to the 6-channel force/moment transducer (Omega IP65, ATI Industrial Automation, Inc.). The force/mo-

\section{TABLE 1: Football helmets studied}

\begin{tabular}{cll}
\hline Type of Helmet/Manufacturer & \multicolumn{1}{c}{ Model } & Mass $(\mathrm{kg})$ \\
\hline varsity helmet* & & \\
Adams & A2000 & 1.40 \\
& A4 & 1.30 \\
\hline Riddell & VSR-4 & 1.90 \\
& Revolution & 1.81 \\
& Revolution IQ & 1.90 \\
& Revolution Speed & 1.87 \\
& Air Advantage & 1.68 \\
& Air XP & 1.77 \\
Schutt & DNA Pro+ & 1.91 \\
& Ion 4D & 1.98 \\
& X1 & 1.97 \\
Xenith & & \\
leather helmet & H-18 & 0.46 \\
Hutch & Air-Lite & 0.80 \\
\hline Wilson &
\end{tabular}

* All met the NOCSAE standard. 
ment transducer defined the global reference frame and was stationary throughout the impact. It is important to note that the NOCSAE headform/Hybrid III neck attachment precluded the use of the standard Hybrid III Model 1706 upper neck load cell and that the neck forces and moments reported here approximate forces at the cervicothoracic junction.

For each struck helmet, the front, oblique front, lateral, oblique rear, and rear directions were impacted at $2.0,3.5$, and $5.0 \mathrm{~m} / \mathrm{second}$ in an attempt to closely match NOCSAE standard contact points on the headform (Figs. 1 and 2 and Table 2). For brevity, only the $5.0-\mathrm{m} / \mathrm{second}$ results are presented here. Each impact direction (5 directions) and velocity (3 velocities) was repeated 3 times for each helmet (13 helmets; 195 total impacts). Head, brain, and neck injury risk was quantified via several parameters: struck headform center of gravity linear acceleration, angular acceleration, angular velocity, GSI, neck force, and neck moment. All data were collected per Society of Automotive Engineers standards. ${ }^{50,51}$ The GSI was calculated from the filtered resultant linear acceleration. Angular acceleration was calculated from the filtered angular velocity derivative. As a secondary quantification, headform center of gravity linear acceleration and angular velocity data were used as inputs to the finite element model SIMon (version 3.051). The outputs from SIMon were calculated risk estimates for DAI, acute subdural hematoma, and brain contusion. . $^{1,25}$

\section{The SIMon Model}

The SIMon model (National Transportation Biomechanics Research Center) consists of a 3D skull and brain
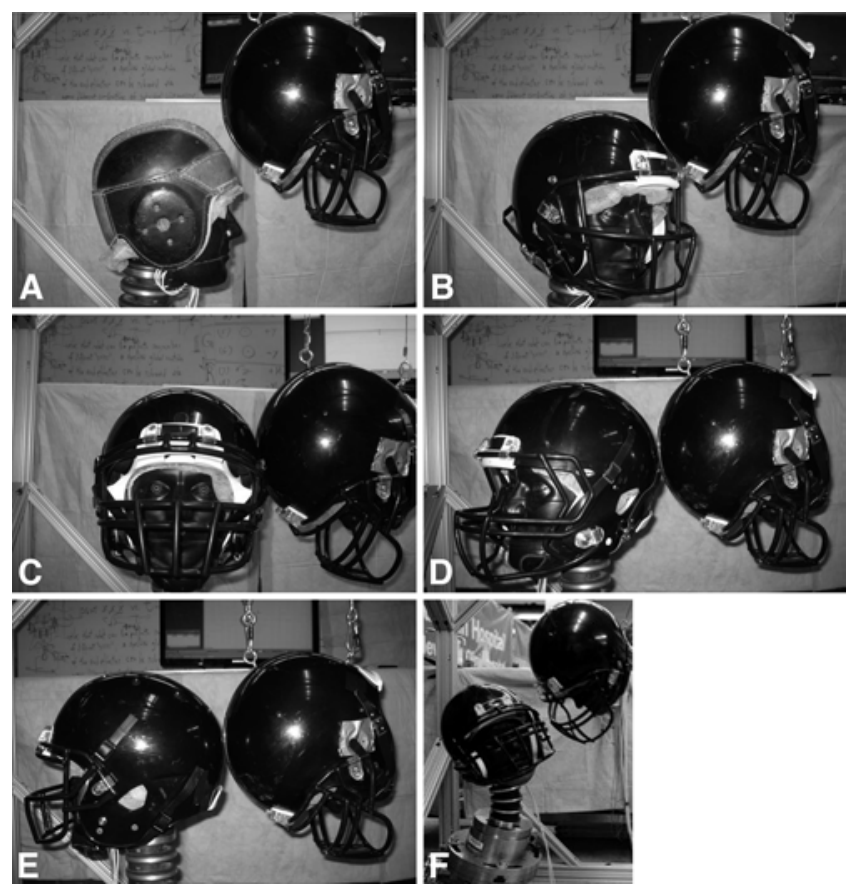

FIG. 1. Photographs showing impact trajectories: front (A), oblique front (B), lateral (C), oblique rear (D), and rear (E). Photograph (F) illustrating lateral neck flexion during impact. with a rigid skull, dura-CSF layer, falx cerebri, whitegray matter, and bridging veins. ${ }^{1,2,52}$ The model represents a 50th percentile male with a head mass of $4.7 \mathrm{~kg}$. There are 10,475 nodes and 7852 elements, with 7776 hexagonal and 76 beam elements. At each time step, SIMon calculates 3 brain injury risk metrics: 1) cumulative strain damage measure (CSDM05), 2) relative motion damage measure (RMDM), and 3) dilatational damage measure (DDM). The CSDM05 is the cumulative percentage of brain volume experiencing $\geq 5 \%$ stretch over the duration of head impact loading. This 5\% stretch value has been correlated with mild DAI (a result of angular brain motion) and transient depolarization. ${ }^{1,2,52}$ The RMDM results indicate the risk of sustaining an acute subdural hematoma due to a bridging vein rupture., ${ }^{1,52}$ More specifically, an RMDM value of 0.5 is equated with an $8 \%$ risk, a value of 1.0 is equated with a $50 \%$ risk, and a value of 2.0 is equated with a $98 \%$ risk of acute subdural hematoma. The DDM, which was developed as an estimate of vacuum contusions within brain tissue, $, 1,25$ was calculated for all impacts.

\section{Football Helmet Standards and the GSI}

Football helmets used in the US must meet standards set by the NOCSAE, ${ }^{38,39}$ which were introduced in 1973. The standards involve dropping a helmeted headform onto an elastomer-padded anvil. The headform is equipped with a 3 -axis linear accelerometer to measure impact acceleration and deceleration only. The $\mathrm{GSI}^{17}$ quantifies severe skull fracture and brain injury risk, not concussion:

$$
\mathrm{GSI}=\int_{0}^{T} \widetilde{a}_{R}(t)^{2.5} d t
$$

where $\tilde{a}_{R}(t)$ is the resultant headform center of gravity acceleration $(\mathrm{g})$ and $[0: \mathrm{T}]$ is the user-defined impact duration (seconds).

To meet the NOCSAE standard, peak GSI must remain below 1200 , equivalent to an approximately $17 \%$ risk of severe skull fracture and brain injury, $25,34,38,39,41,42,44,53$ as quantified by the AIS (Table 3$)^{18}$ and post-World War II head impact studies in humans and animals. ${ }^{17}$ The NOCSAE standard GSI threshold is well in excess of the values associated with concussion, ${ }^{24,55}$ and all varsity helmets in use today vastly outperform the NOCSAE threshold.

\section{Results}

We tested the head impact protectiveness of 11 varsity helmets meeting the NOCSAE standard and 2 early 20th century leather helmets. Helmet-to-helmet impacts produced near- and subconcussive head impact doses approximately as severe as the 95th percentile of on-field collegiate and high school impacts, while impact energy and momentum were on par with NOCSAE standards. For many of the impacts, leather helmets demonstrated head impact doses and injury risks on par with or better than those demonstrated by several of the varsity helmets. Overall, the majority of impact dose results indicated a 


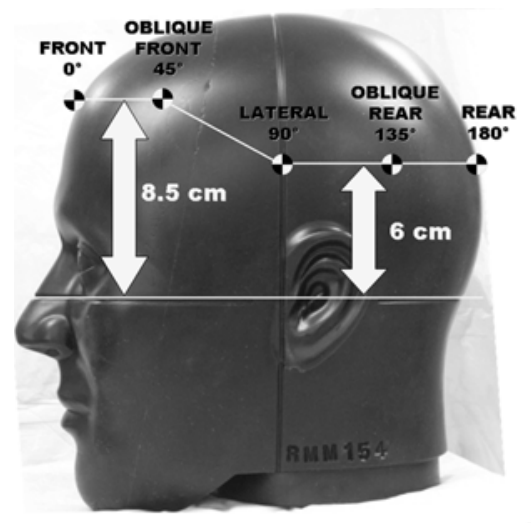

A
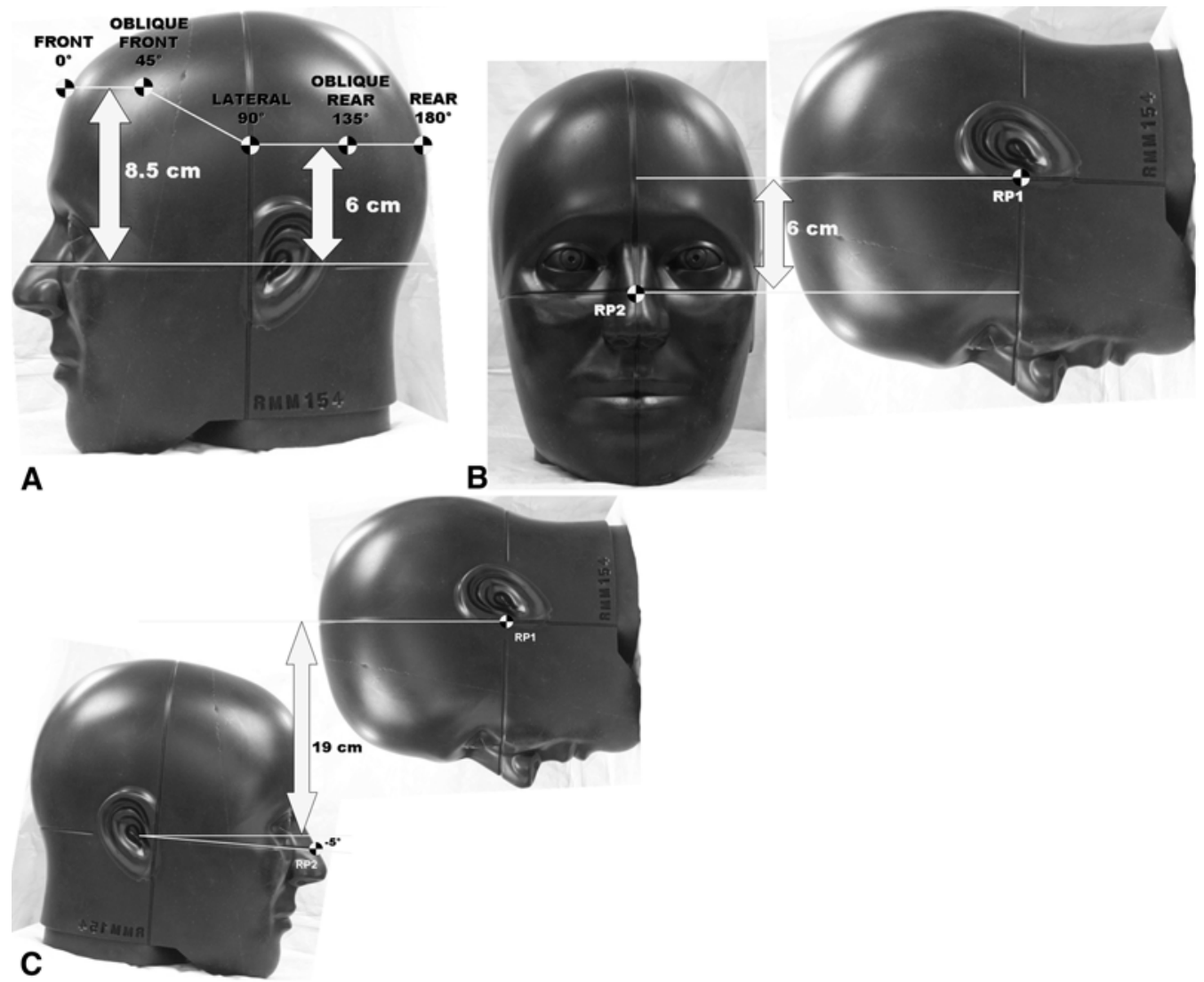

FIG. 2. Image (A) depicting NOCSAE standard contact points on a struck headform. Image (B) depicting the lateral, oblique rear, and rear impact vertical distance from reference point 1 (RP1) to RP2. Image (C) depicting the front and oblique front impact vertical distance from RP1 to RP2. Elevation $=-5^{\circ}$.

similar protectiveness profile for the leather helmet and the modern varsity helmets as regards impacts in the near- and subconcussive range studied.

As shown in Fig. 3, traces for mean linear acceleration/time, mean angular acceleration/time, mean angular velocity/time, mean neck force/time, and mean neck moment/time for the leather helmets were similar or less severe than those for several of the varsity helmets.

When examining the mean of maximum values alone (Fig. 4), the linear acceleration, angular acceleration, angular velocity, GSI, neck force, and neck moment results for the leather helmet were on par with those for many of the varsity helmets.

After analyzing the impact response data, both the mean linear acceleration and mean angular velocity responses for each set of varsity and leather helmet impacts

\section{TABLE 2: Helmet impact orientation}

\begin{tabular}{lccc}
\hline & & & Vertical Distance \\
Impact Direction & Azimuth $\left(^{\circ}\right)$ & Elevation $\left(^{\circ}\right)$ & RP1 to RP2 $(\mathrm{cm})$ \\
\hline front & 0 & -5 & 19 \\
oblique front & 45 & -5 & 19 \\
lateral & 90 & 0 & 6 \\
oblique rear & 135 & 0 & 6 \\
rear & 180 & 0 & 6 \\
\hline
\end{tabular}

were used as inputs to drive the SIMon finite element brain injury risk prediction model (Fig. 5A and B). Considering the CSDM05 results shown in Fig. 5C, one of the leather helmets had the lowest DAI risk of all the varsity and leather helmets tested. In Fig. 5D the RMDM results indicated

\section{TABLE 3: Abbreviated Injury Scale*}

\begin{tabular}{cl}
\hline Rating & \multicolumn{1}{c}{ Description } \\
\hline 0 & no injury \\
1 & minor injury \\
2 & moderate injury \\
3 & serious injury \\
4 & severe injury \\
5 & critical injury \\
6 & maximal; currently untreatable \\
\hline
\end{tabular}

* The scale, from a minimum of 0 to a maximum of 6 , contains integer values that are included as a decimal value at the end of any injury coded. Subacute concussion would be awarded an AIS score of 0 . Although simplistic in nature, the rating system bases injury scaling on several dimensions of severity: threat to life, mortality (theoretical, expected, and actual), amount of energy dissipated or absorbed, hospitalization and need for intensive care, duration of hospital stay, treatment cost, treatment complexity, duration of treatment, temporary and permanent disability, permanent impairment, and quality of life. 
A. Bartsch et al.
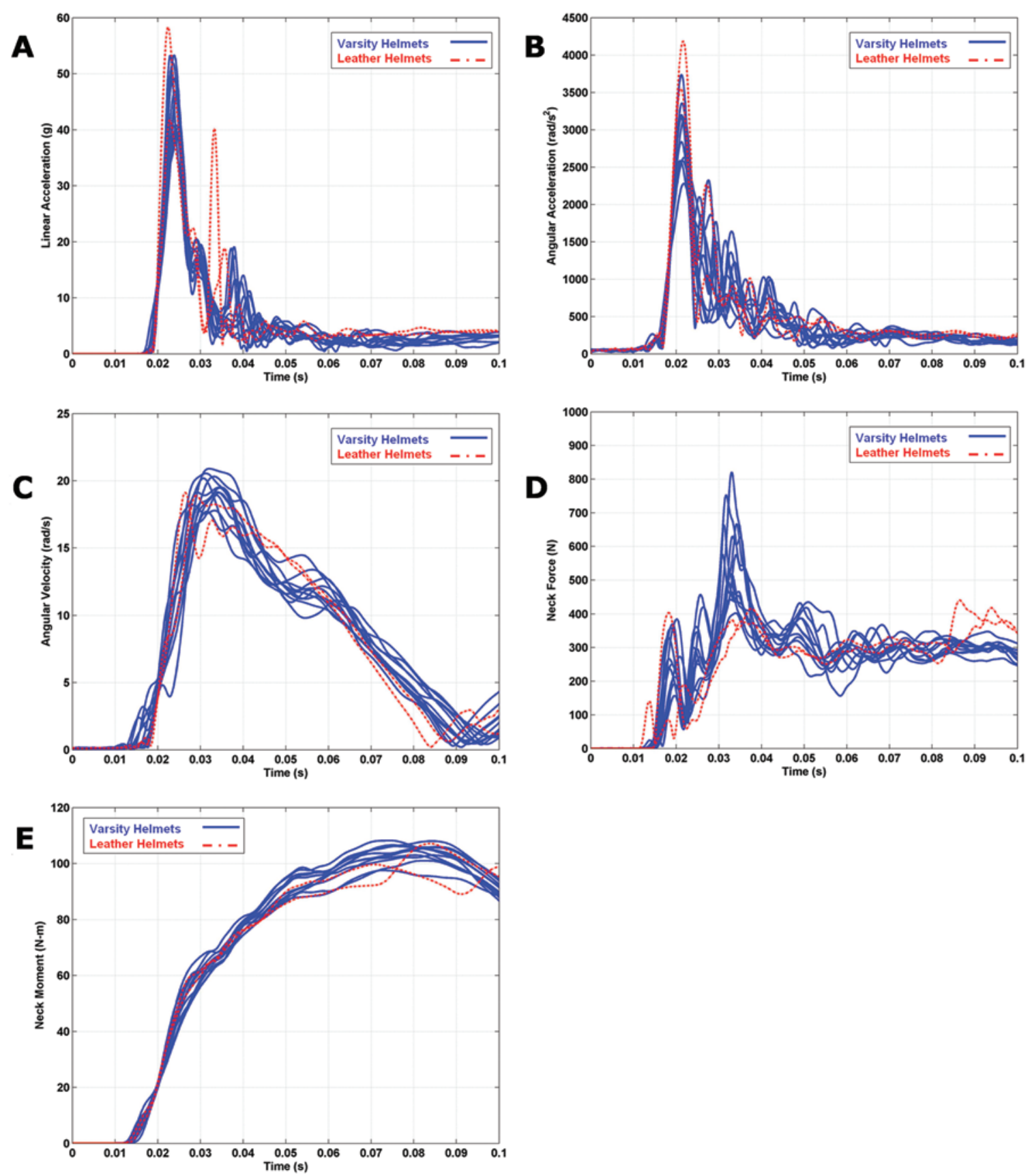

Fıg. 3. Sample response data collected for 11 varsity helmets and 2 leather helmets. Graphs showing resultant linear acceleration/time curves (front, A), angular acceleration/time curves (oblique front, B), angular velocity/time curves (rear, C), neck force/time curves (oblique rear, D), and neck moment/time curves (lateral, E).

that the leather helmets were associated with a risk of acute subdural hematoma on par with the varsity helmets. The DDM results for both leather and varsity helmets were negligible in all impacts and thus were omitted.

\section{Discussion}

\section{Concussion: A Clinical Perspective}

From a clinical perspective, the mechanism of concussion has been theorized to result from an inertial, impulsive, and/or blunt trauma with acceleration and/or de- celeration energy transmitted either directly to the head or indirectly to the head through the torso and spine. The mechanisms by which concussion manifests are controversial and most likely multifactorial. Regardless of the actual injury mechanisms at the cellular level, concussion typically leads to the rapid onset of short-lived impairment of neurological function that resolves spontaneously. Symptom resolution may not be indicative of injury resolution. Any period of an observed or self-reported symptom complex, including transient confusion, disorientation, impaired consciousness, dysfunction of memory, or 

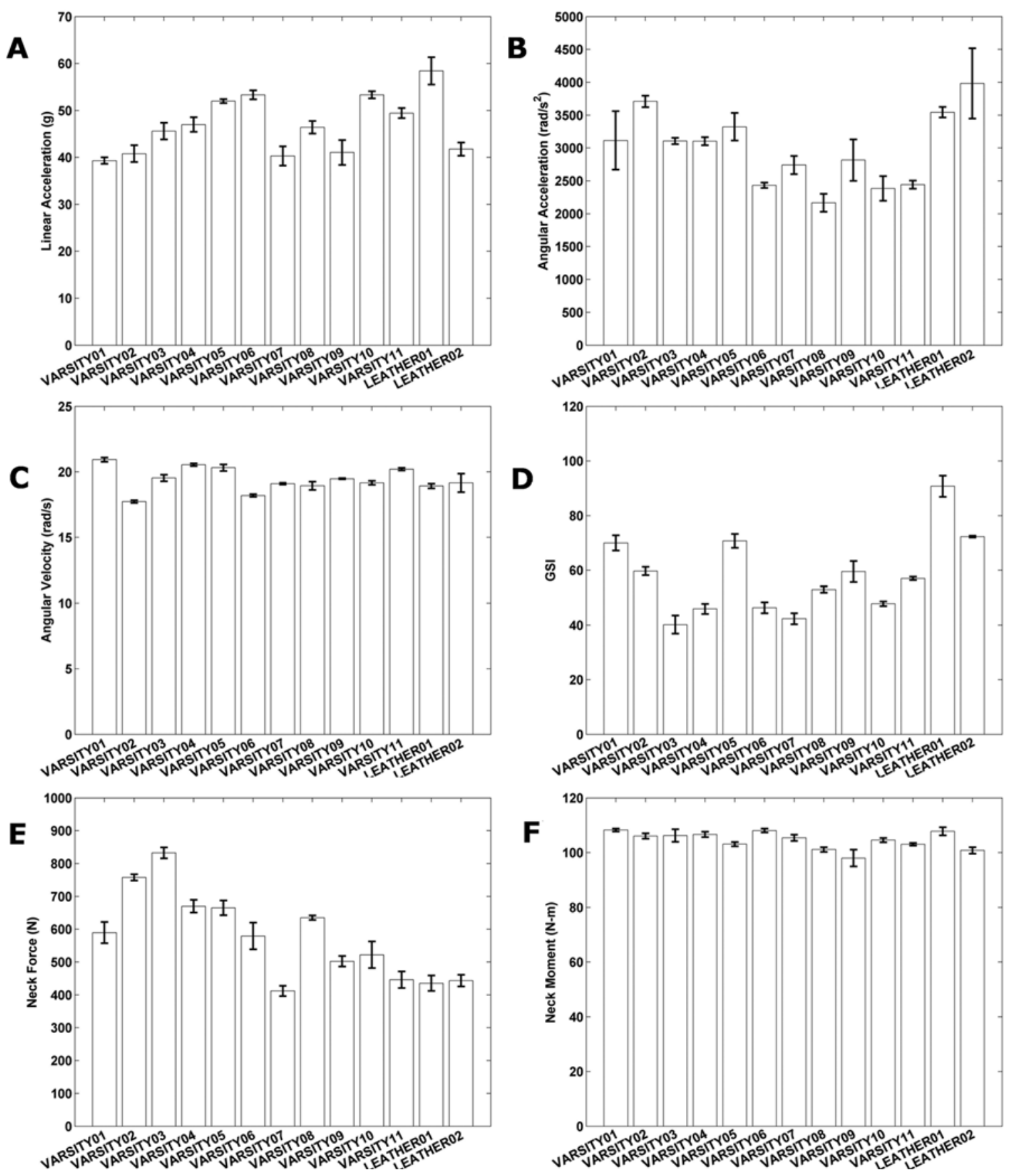

FIG. 4. Bar graphs showing sample response data maxima: linear acceleration (front, A), angular acceleration (oblique front, B), angular velocity (rear, C), GSI (lateral, D), neck force (oblique rear, E), and neck moment (lateral, F). Error bars indicate \pm 1 SD.

loss of consciousness lasting less than 30 minutes, may occur. Concussion can lead to neuropathological changes, but the acute clinical symptoms largely reflect functional disturbances rather than structural injuries. Concussive impacts are typically associated with grossly normal structural neuroimaging studies. Functional disturbances may include observed signs of neurological or neuropsychological dysfunction, headache, dizziness, irritability, fatigue, or poor concentration. Resolution of the clinical and cognitive symptoms usually follows a typical course.
Symptoms may be prolonged or persistent and may lead to permanent brain damage and diseases, symptoms, or conditions thought to be caused by impact dose accumulation. Examples of the latter include chronic traumatic encephalopathy, neuropsychological deficits, dementia pugilistica, second impact syndrome, depression, sleep disorders, difficulty concentrating, memory problems, headaches, dizziness, irritability, inability to perform daily living activities, chronic disability, Alzheimer disease, and Parkinson disease. $6,21,22,31,33,37,43,45$ 

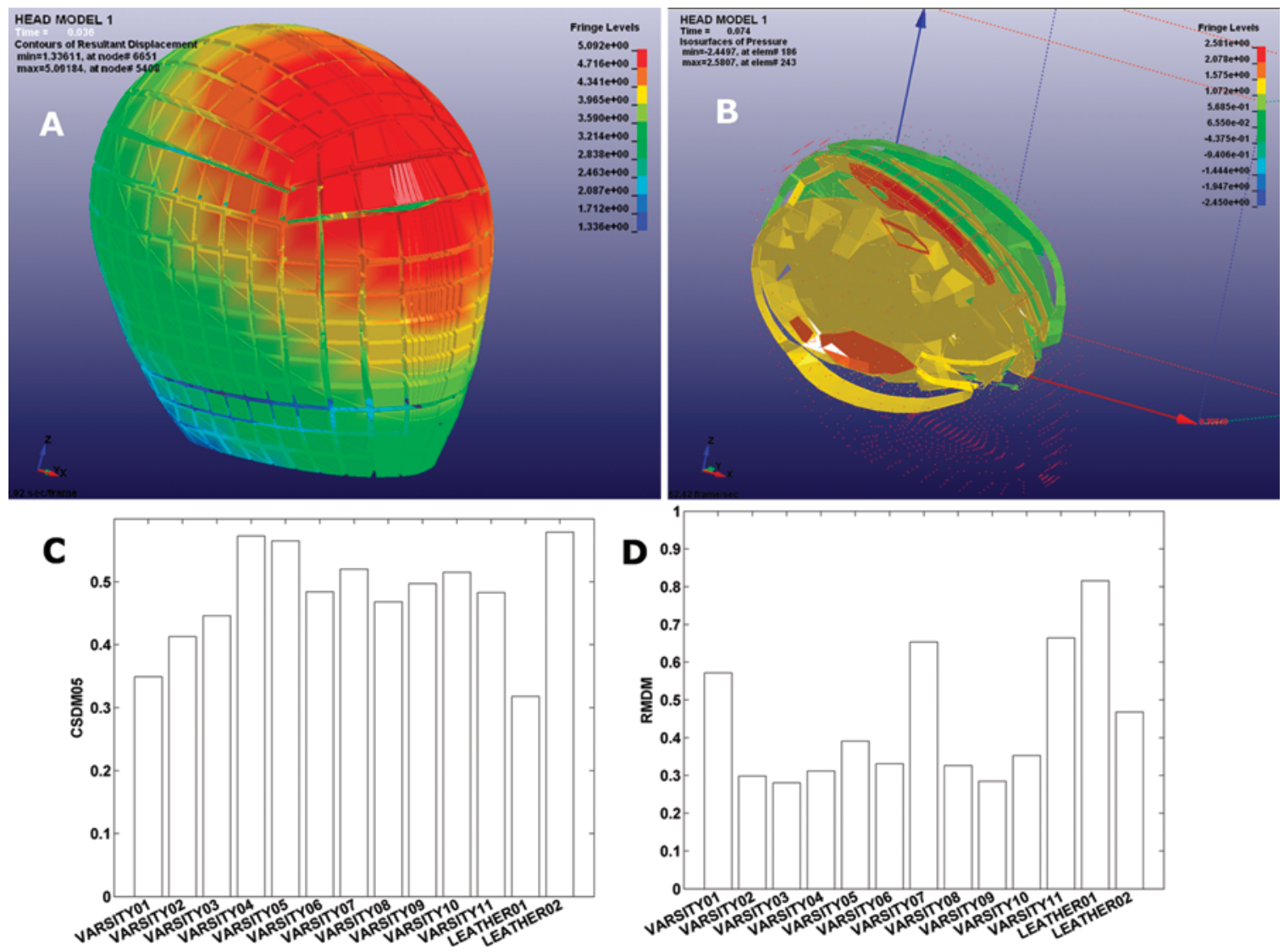

FIG. 5. The SIMon finite element brain injury model (A and B) was used to calculate theoretical brain injury results for CSDM in an oblique rear direction (C) and RMDM in a rear direction (D).

\section{Historical Review of American Football and Head Injury}

Modern varsity football helmets, like that shown in Fig. 6, have been developed and refined over the course of the past 2 centuries in response to on-field injuries, rule changes, and injury reduction strategies. As shown in Fig. 7 , football and injury risk in football have a storied history in the US dating back to the first game between Princeton and Rutgers on November 8, 1869. Around the turn of the century, football was becoming known as much for violence as for play on the field. This led innovators to reduce injury risk through various measures, including the prohibition by law of the famed "flying wedge" formation (1893), the introduction of the first leather headgear (1896), the endorsement by President Roosevelt of safer rules of play to address the 18 on-field fatalities in 1905 , and the institution of the forward pass (1906). Innovations post-World War I included the formation of the NFL (1922), the development of the first face mask (1935), and the patenting of the first plastic helmet by Riddell (1940). During the 1940s, several authors published the initial pioneering data on head impact tolerance and cerebral concussion related to linear acceleration. In these studies, however, "cerebral concussion" referred to a direct insult to the skull and brain causing obvious, macroscopically visible severe injury as opposed to the 21 st century description of cerebral concussion causing no visible skull or brain injury. In 1948, among concerns that hard-shell helmets increased injury risk, the NFL banned hard-shell helmets for 1 year. By 1956, the first face mask penalties were instituted in the NFL to protect players' heads and necks. By 1966, rotational head injury theories were proposed, linking brain tissue shearing to dysfunction. Also in 1966, Gadd developed a head injury severity index, the GSI, based on acceleration during head impact.

In 1969, 38 football-related fatalities occurred na-

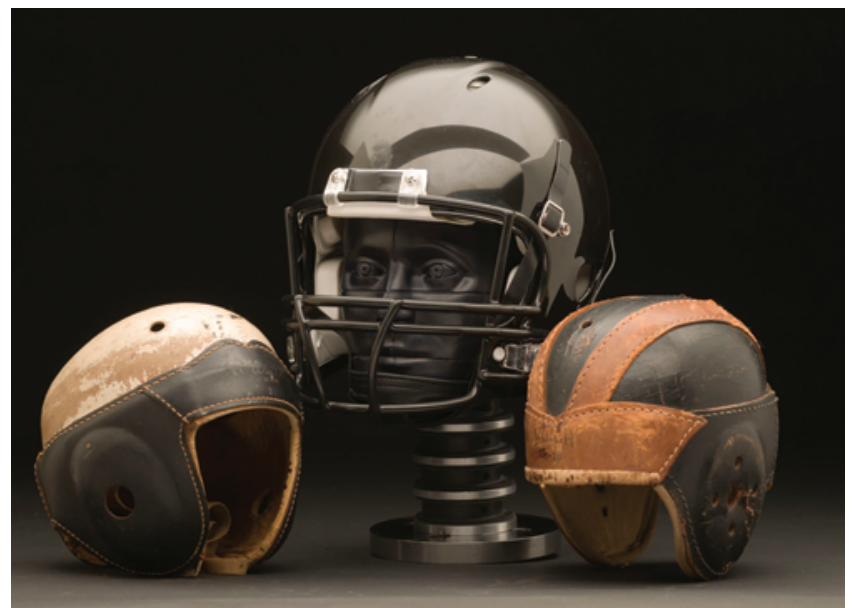

Fig. 6. Photograph of 21 st century varsity football helmet (center), which may be technologically advanced, but do these helmets always provide greater protection than early 20th century leather headgear, or leatherheads (left and right)? 

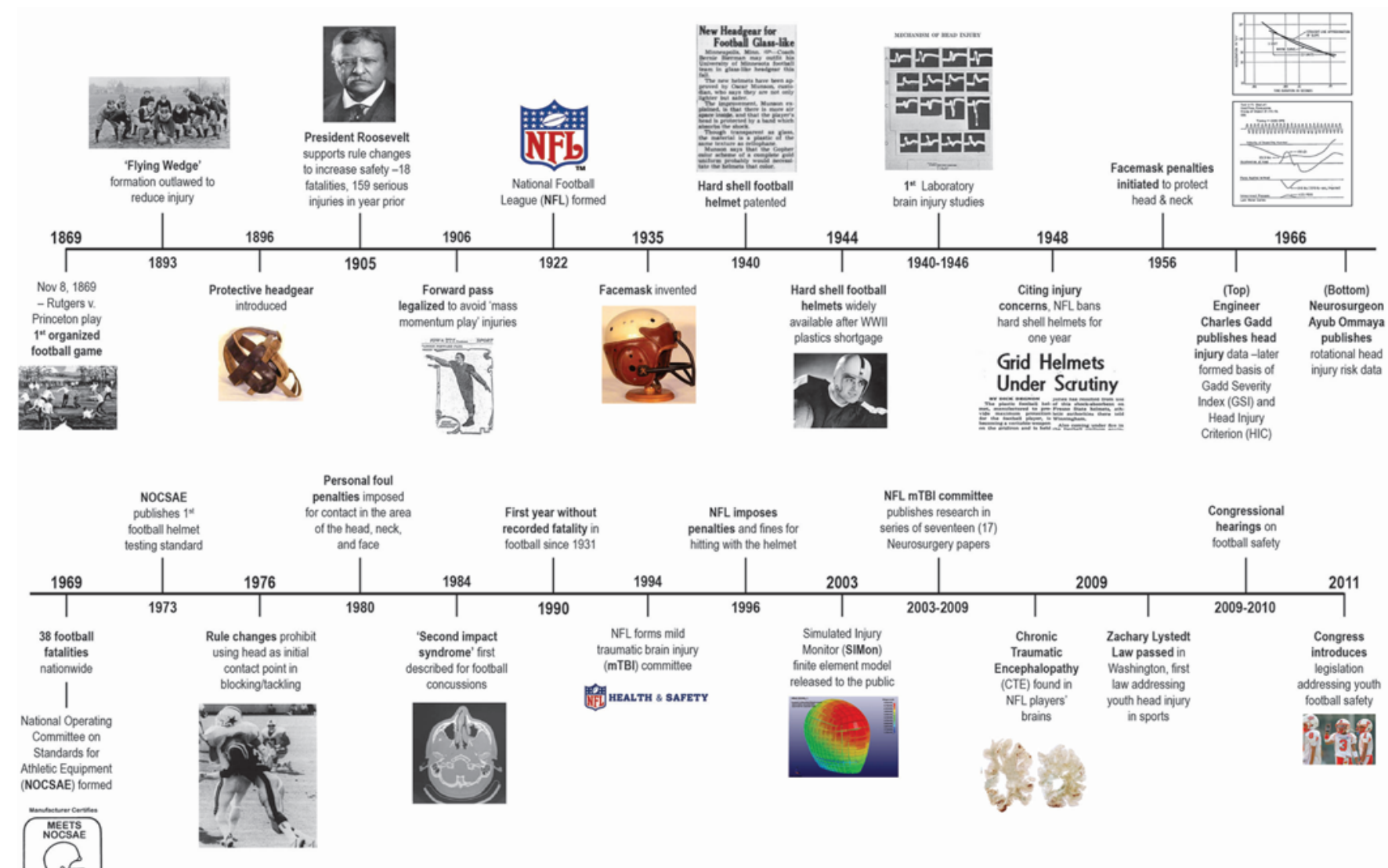

FIG. 7. Timeline of historical highlights of American football and injury relationship.

tionwide. As a response to this appalling statistic, the NOCSAE was formed in 1969. It established the first football-helmet testing standard in 1973, using the GSI. In further efforts to reduce severe injuries, the pioneering work of Dr. Joseph Torg and the NOCSAE contributed to rule changes in 1976 that outlawed using the head as the initial blocking or tackling contact point. In 1980, further rule changes placed greater restrictions on contact with the head, neck, and face, with personal foul penalties being instituted for striking, swinging, or clubbing these sensitive areas. In 1984, the football-related second impact syndrome, that is, the risk of suffering a devastating head injury when returning to play before a first head injury has fully healed, was first posited in the medical literature. These advances in understanding catastrophic football injuries and rule changes culminated in a fatality-free season in 1990.

In the 1990s, concern was mounting regarding what was described as mild TBI. In 1994, the NFL established the Mild Traumatic Brain Injury Committee to investigate head injury in football. By 1996, the NFL instituted personal foul penalties and monetary fines for hits with the helmet or to the head of an opponent. By 2003, the first finite element model to quantify skull and brain injury risk was made available to the research community via the SIMon program. Brain injury risk simulation tools and innovative traumatic neuromechanics research played key roles in the 2003-2009 publications of sev- eral well-known laboratory impact studies that simulated on-field NFL collisions. By 2009, heightened awareness regarding concussion and the long-term sequelae associated with repeated concussive and subconcussive brain injuries had emerged. The level of awareness was further escalated by the well-publicized passage of the Zackery Lystedt law in Washington State, the first law to specifically address concussion in youth sports. That same year, a seminal study was published regarding chronic traumatic encephalopathy, a type of progressive brain deterioration found in the autopsied brains of football players and several other ex-athletes with a history of head impact dose accumulation over years. Congressional hearings on safety in football were held in 2009 and 2010 amid a rash of media coverage on the decline and deaths of several high-profile athletes with a history of head impacts. Finally, in 2011, bills were introduced in the Senate and House of Representatives that addressed adult helmet safety standards, moved to establish youth helmet safety standards, and proposed the investigation of potentially deceptive advertising language pertaining to helmet-related concussion protection.

\section{The GSI}

Although severe head and neck injuries were greatly reduced $^{36}$ after the NOCSAE football helmet standards and rule changes were instituted in 1970s and 1980s, the latest data indicate that the incidence of concussion is 
climbing. ${ }^{19}$ One possible reason for the increase in concussions is that the NOCSAE GSI threshold is no longer a state-of-the-art injury predictor. The GSI has long been known to have major deficits when applied to the padded-head impacts in football. A prime example can be found from the early 1970s: a bare-head impact duration of $<15$ msecs was "considered safe as far as cerebral concussion is concerned." ${ }^{27}$ But based on published onfield data, ${ }^{11-14,46,47}$ we now know that clinically symptomatic concussions have resulted from impacts lasting less than 15 msecs. Furthermore, while on-field impacts can have a characteristically shaped acceleration curve that is similar to that for an NOCSAE test (Fig. 8), the NOCSAE test curves have a widened pulse width and longer duration that are not indicative of head impacts on the field of play. Additionally, the Head Injury Criterion, ${ }^{54}$ a GSI derivative, was declared insufficient as a helmet standard injury risk metric years ago, ${ }^{15}$ and preeminent researchers have called for an end to its use as a head injury risk quantifier. ${ }^{32}$ All of these findings bring into question the on-field protectiveness translation and clinical relevance of the GSI as the sole football head injury risk metric in use by the NOCSAE.

\section{Modern Football Concussion Studies}

Interest in concussion escalated exponentially in the 1990s and was initiated in large part by the highly publicized and premature retirements of notable professional US football players, including Merril Hoge and Al Toon, due to the cumulative adverse effects of concussion. In response, the NFL proactively formed the Mild Traumatic Brain Injury Committee in 1994 (currently the NFL Head, Neck and Spine Medical Committee) to better understand NFL player head impact dose and traumatic neuromechanics. First published in 2003, several elegant laboratory experiments used instrumented crash test dummies and "newer" (for example, varsity) helmets

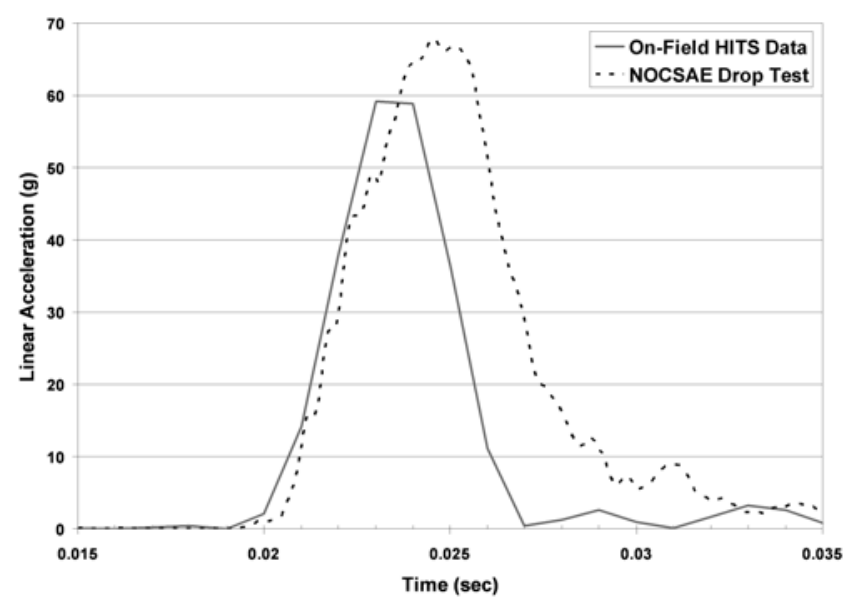

FIG. 8. Graph showing a comparison between a single varsity helmet (Riddell VSR-4) in a lateral on-field impact from a Head Impact Telemetry System (HITS, solid line) and NOCSAE drop testing (dashed line). On-field impact measured $59.2 \mathrm{~g}$ of peak resultant linear acceleration. The NOCSAE drop test was conducted at $3.0 \mathrm{~m} / \mathrm{second}$ (18-in drop height) and registered $67.7 \mathrm{~g}$ of peak resultant linear acceleration. Note that the curves have a similar shape, but the line representing the NOCSAE drop test has a widened pulse width and longer time duration. in very high impact dose NFL in-game head impact reconstruction studies. ${ }^{41,42,55}$ Peak linear head accelerations in the range of $50 \mathrm{~g}-150 \mathrm{~g}$ were produced. These studies led to the conclusion 55 that "with newer (varsity) helmets, there was a trend toward 10 to $20 \%$ lower risks of concussion." But "lower risks of concussion" were defined solely by severe skull fracture and brain injury risk, which is inconsistent with the current clinical and neuromechanical definition, as described above.

In another study published in 2006 and funded by the football helmet maker Riddell, among 2141 high school football players wearing the varsity Riddell Revolution model and "standard" helmets, researchers determined that "the Revolution helmet was associated with approximately a $31 \%$ decreased relative risk and a $2.3 \%$ decreased absolute risk for sustaining a concussion." $8 \mathrm{Au}$ thors of this study also concluded that the published NFL mild TBI studies have "led to changes in helmet design and new helmet technology, which appear to have beneficial effects in reducing the incidence of cerebral concussion in high school football players." Note that the validity of this study's results were challenged on the basis of methodological flaws, bias, and conflicts of interest. ${ }^{9}$

Recently, instrumented varsity Riddell Revolution football helmets were used in high school and collegiate competitions. A mean calculated center of gravity acceleration of $22 \mathrm{~g}-32 \mathrm{~g}$ was found for on-field impacts. $5,12,20,35$ These data strongly suggested that routine on-field head impact doses in high school and college were in the subconcussive range as compared with the aforementioned NFL studies of $50 \mathrm{~g}-150 \mathrm{~g}$ impacts. ${ }^{41,42,55}$

\section{Our Study}

The pre-World War II vintage leather helmets in our tests, despite their lack of technologically advanced energy-absorbent materials, frequently were associated with head impact doses and theoretical injury risks that, based on linear acceleration, angular acceleration, angular velocity, neck force, and neck moment measures, were similar to or lower than those for several 21st century varsity helmets in near- and subconcussive impacts. More surprisingly, although varsity helmets have been optimized to meet the linear acceleration-based GSI head injury risk criterion, ${ }^{17}$ our results indicated that the vintage leather helmets allowed comparable linear acceleration performance in the front and oblique front impact directions. The leather helmets also performed surprisingly well as compared with modern varsity helmets in terms of angular acceleration, angular velocity, neck force, and neck moment responses in nearly all conditions except lateral angular acceleration. Even in cases in which the varsity helmets were associated with superior angular responses, these differences in our near- and subconcussive impacts were not associated with a meaningful reduction in head injury risk based on current data. . $2,17,20,26,27,29,34,40,44,52,54^{2}$

Using the SIMon finite element model, ${ }^{52}$ brain injury risk metrics relating impact dose to DAI (CSDM05) and acute subdural hematoma (RMDM) indicated that in many cases the leather helmets outperformed varsity helmets. Even the potential theoretical risk of brain contusions due to vacuum pressure, as measured by the DDM, in the 
leather helmets was similar to that for the varsity helmets, that is, near 0 in all impacts. In short, while the impact dose for the leather helmet exceeded that for some of the varsity helmets in a few specific impact-testing scenarios (for example, lateral linear acceleration, oblique rear linear acceleration, rear linear acceleration, and lateral angular acceleration), in the majority of our impacts the impact dose for the leather helmet was equal to or less than that for the varsity helmets. Hence, we propose that head injury risk reduction with the leather helmet was quantifiably and probably clinically comparable to that with the varsity helmets for the near- and subconcussive impact doses for most of the dosage parameters studied. These findings were in stark contrast to those in published works touting the " 10 to $20 \%$ lower risks" 55 and " $31 \%$ decreased relative risk" of concussion when using 21 st century varsity helmets in comparable dose on-field impacts.

Our results may be related in part to the near- and subconcussive helmet-to-helmet impact doses we utilized. Our impact doses replicated common on-field nearand subconcussive impact doses up to approximately the 95th-percentile severity. Therefore, unlike higher-dose concussive NFL varsity helmet studies ${ }^{41,42,55}$ or helmetanvil impact tests to quantify severe skull fracture and brain injury, ${ }^{38,39}$ the impacts studied here probably did not appreciably engage the varsity helmet impact-attenuating systems that have been optimized for NOCSAE helmetanvil tests. Thus, while the leather helmets offered little in the way of modern energy-absorbing technology, varsity helmet stiffness may have provided less than optimal energy absorbency in the common near- and subconcussive impacts we studied. Based on the impacts tested, it appears that much could be gained in terms of future varsity helmet designs by developing strategies that minimize linear and rotational head impact dose and dose accumulation in these most common on-field impact scenarios.

Finally, our findings provide preliminary insights into the lower head impact energy and momentum dose currently endured by youth football players wearing helmets similar to the modern varsity helmets studied here. These are very important considerations for the future of football, especially as they apply to the more than 3 million youth players in the US (http://usafootball.com/sites/ default/files/USA_Football_one_pager_Dec_2010.pdf). Currently, there exists no head or neck injury risk standard specifically tailored for youth football helmets, and unlike youth baseball with its discrete pitch counts aimed at preserving developing upper extremities, there is no cap on the number of head impacts sustained during practices and games played by youths with developing neurological systems. In youth football, in which children as young as 6 years of age regularly incur head impacts, injury risk standards and limits on head impact doses are desperately needed to minimize short-term acute head injury risk and long-term risk due to dose accumulation. Recent legislation introduced in the US Congress serves as notice regarding the critical nature of the need for these safety standards. ${ }^{7,8}$ Additionally, helmets used in youth football have historically been scaled-down "little adult" versions of varsity helmets. Therefore, scaling down varsity football helmets fails to consider youth players' impact condi- tions, anthropometry, developing spinal musculature, maturing brain, and head-neck size disparity. And thus, today's youth helmets may have energy-absorbing features that are not optimized for these lower-dose impacts. Our findings provide reasons to further explore this theory.

\section{Study Weaknesses}

This study had several intrinsic weaknesses. First, pendulum impact testing involved the use of a custom impact setup requiring modification of the NOCSAE headform to attach it to the Hybrid III neck as well as the removal of some material from the headform "mouth" to rigidly affix the angular velocity sensors to the headform. This attachment may have over-compressed the Hybrid III neck rubber nodding blocks. However, because the NOCSAE drop testing standard ${ }^{38,39}$ was the only standard means of quantifying football helmet protectiveness and because this standard utilized a helmeted headform without a face mask, there was no standard impact setup that incorporated the neck or angular velocity measurement. Therefore, although our impact setup was nonstandard, it was designed solely to conduct highly controlled horizontal impacts with energy similar to that in the NOCSAE standard ${ }^{38,39}$ while providing added monitoring of additional injury risk parameters.

Second, we did not include face mask impacts in our testing paradigm. The face mask has been shown to be a common contact point and to have potential influence on concussive injury risk. ${ }^{41,42,55}$ Nevertheless, face mask impacts were not assessed because of the chaotic nature and high variability found in prior face mask impact studies.

Third, we studied only a limited number of helmets and impact combinations. We tested, through a limited combination of impact direction and dosage, a single sizelarge helmet (each with 1 type of face mask and chinstrap) of each of the 11 varsity models meeting the NOCSAE football helmet standard. Because of potential variability among same-model helmets, a statistically relevant population of each helmet make, model, face mask, and chinstrap may need to be studied under a variety of additional impact scenarios for a complete impact response data set. Regardless, to determine statistically relevant sample sizes for each helmet, one must consider that there were literally infinite numbers of football helmet impact conditions from which to choose. Therefore, we picked our highest priority near- and subconcussive impact conditions but recognized that many more tests than those conducted here would be required to more fully understand this complex domain. The small sample size used here should be expanded to accurately determine intrahelmet variability and its effects on testing results.

\section{Future Work}

Helmet fit and influence on injury risk metrics must be studied in the future. Helmet manufacturers encourage players to wear a snug-fitting helmet for proper onfield competition as well as for injury risk mitigation. And in our study, all helmets tested were snugly fitted per the qualitative description within the NOCSAE football helmet standard. ${ }^{38,39}$ But snug-fitting helmets might not necessarily minimize injury risk. There may have been 
an advantage to helmet padding with an increased compliance at the padding-skin interface to allow for more energy absorption; however, this was not tested here. Moreover, strategies, such as the use of a semisoft energy-absorbing shell, viscoelastic padding, or breakable helmet components (such as styrene foams inserted into automotive bumper systems), may have provided added protection via reduced energy transmission to the brain. Furthermore, the effect of potential degradation of helmet shell integrity due to solvent-substrate interactions during helmet reconditioning should be investigated. If reconditioned helmet painting did degrade the polymer shell, it would probably influence helmet protection. Finally, future studies on helmet standards as well as on varsity and youth football helmets must be conducted to promulgate helmet outer shell, interior padding, and face mask design strategies that minimize both acute concussion risk caused by single-impact dosage and long-term brain injury risk due to subconcussive impact dosage accumulation.

\section{Conclusions}

Our findings indicated that varsity helmets, which have been optimized toward high-severity linear acceleration dose attenuation, frequently did not provide substantial protective superiority in near- and subconcussive head impacts as compared with leather helmets. Helmet manufacturers must consider strategies that minimize high-severity dose impacts (for example, severe skull fracture and brain injury) as well as lower-severity dose impacts (for example, acute concussion and clinically silent subconcussive impacts).

Furthermore, the data presented here strongly suggest that the GSI, which rates helmet protectiveness based solely on the risk of severe skull fracture, is insufficient as a stand-alone concussion or near-concussive injury risk metric. Hence, the GSI should no longer be used as the sole metric for assessing football helmet protectiveness. There currently exist voluminous in vivo head impact, finite element modeling, and crash test dummy data in the peer-reviewed literature that can serve as a strong foundation from which statistically and experimentally sound 21st century head and neck injury risk metrics for American football helmets could be rapidly developed. The results of our study provide a rationale for doing so.

We believe that American football governing bodies should mitigate head and neck injury risk by limiting impact dose accumulation. Furthermore, we believe that American football helmets should be designed to minimize high-severity dose (fracture and brain injury) and low-severity dose (concussive and subconcussive) impacts. Finally, we believe that youth helmet testing standards, which would have a great effect on youth helmet protectiveness, must be rapidly advanced to minimize head impact dose as well as short- and long-term head and neck injury risk in maturing athletes.

\section{Disclosure}

The authors report no conflict of interest concerning the materials or methods used in this study or the findings specified in this paper.

Author contributions to the study and manuscript prepara- tion include the following. Conception and design: all authors. Acquisition of data: all authors. Analysis and interpretation of data: all authors. Drafting the article: all authors. Critically revising the article: all authors. Reviewed submitted version of manuscript: all authors. Approved the final version of the manuscript on behalf of all authors: Bartsch. Statistical analysis: Bartsch, Prakash. Study supervision: Bartsch.

\section{References}

1. Bandak FA, Eppinger R: A Three-Dimensional Finite Element Analysis of the Human Brain Under Combined Rotational and Translational Accelerations. SAE International Website. (http://papers.sae.org/942215/) [Accessed September 27, 2011]

2. Bandak FA, Zhang AX, Tannous RE, DiMasi F, Masiello P, Eppinger R: SIMon: A Simulated Injury Monitor; application to head injury assessment. SAE International Website. (http:// papers.sae.org/2001-06-0222) [Accessed September 27, 2011]

3. Battista J: N.F.L. fines players for hits to head. New York Times. October 19, 2010

4. Battista J: N.F.L. moves kickoffs to the 35. New York Times. March 23, 2011 (http://www.nytimes.com/2011/03/23/sports/ football/23rules.html) [Accessed September 18, 2011]

5. Broglio SP, Schnebel B, Sosnoff JJ, Shin S, Fend X, He X, et al: Biomechanical properties of concussions in high school football. Med Sci Sports Exerc 42:2064-2071, 2010

6. Cantu RC: Second-impact syndrome. Clin Sports Med 17: 37-44, 1998

7. Children's Sports Athletic Equipment Safety Act, HR 1127, $112^{\text {th }}$ Congress (2011-2012) (http://www.govtrack.us/congress/ bill.xpd?bill=h112-1127) [Accessed September 27, 2011]

8. Children's Sports Athletic Equipment Safety Act, S 601, 112th Congress (2011-2012) (http://www.govtrack.us/congress/bill. xpd?bill=s112-601) [Accessed September 27, 2011]

9. Collins M, Lovell MR, Iverson GL, Ide T, Maroon J: Examining concussion rates and return to play in high school football players wearing newer helmet technology: a three-year prospective cohort study. Neurosurgery 58:275-286, 2006

10. Concussion Treatment and Care Tools Act of 2010, HR 1347, $111^{\text {th }}$ Congress (2009-2010) (http://www.govtrack.us/congress/ bill.xpd?bill=h111-1347) [Accessed September 27, 2011]

11. Crisco JJ, Fiore R, Beckwith JG, Chu JJ, Brolinson PG, Duma $\mathrm{S}$, et al: Frequency and location of head impact exposures in individual collegiate football players. J Athl Train 45:549559,2010

12. Duma SM, Manoogian SJ, Bussone WR, Brolinson PG, Goforth MW, Donnenwerth JJ, et al: Analysis of real-time head accelerations in collegiate football players. Clin J Sport Med 15:3-8, 2005

13. Duma SM, Rowson S: Every Newton Hertz: a macro to micro approach to investigating brain injury. Conf Proc IEEE Eng Med Biol Soc 2009:1123-1126, 2009

14. Duma SM, Rowson S: Past, present, and future of head injury research. Exerc Sport Sci Rev 39:2-3, 2011

15. Fenner H Jr, Thomas DJ, Gennarelli T, Pintar FA, Becker EB, Newman JA, et al (eds): Final Report of Workshop on Criteria for Head Injury and Helmet Standards. Milwaukee WI: Medical College of Wisconsin and Snell Memorial Foundation, 2005

16. Finkelstein EA, Corso PS, Miller TR: The Incidence and Economic Burden of Injuries in the United States, 2000. New York: Oxford University Press, 2006

17. Gadd CW: Use of weighted-impulse criterion for establishing injury hazard. SAE International Website. (http://papers. sae.org/660793/) [Accessed September 27, 2011]

18. Gennarelli TA, Wodzin E (eds): Abbreviated Injury Scale. Barrington IL: Association for the Advancement of Automotive Medicine, 2005

19. Gessel LM, Fields SK, Collins CL, Dick RW, Comstock RD: Concussions among United States high school and collegiate athletes. J Athl Train 42:495-503, 2007

20. Greenwald RM, Gwin JT, Chu JJ, Crisco JJ: Head impact 
severity measures for evaluating mild traumatic brain injury risk exposure. Neurosurgery 62:789-798, 2008

21. Guskiewicz KM, Marshall SW, Bailes J, McCrea M, Cantu $\mathrm{RC}$, Randolph C, et al: Association between recurrent concussion and late-life cognitive impairment in retired professional football players. Neurosurgery 57:719-726, 2005

22. Guskiewicz KM, Marshall SW, Bailes J, McCrea M, Harding HP Jr, Matthews A, et al: Recurrent concussion and risk of depression in retired professional football players. Med Sci Sports Exerc 39:903-909, 2007

23. Guskiewicz KM, Weaver NL, Padua DA, Garrett WE Jr: Epidemiology of concussion in collegiate and high school football players. Am J Sports Med 28:643-650, 2000

24. Higgins M, Halstead PD, Snyder-Mackler L, Barlow D: Measurement of impact acceleration: mouthpiece accelerometer versus helmet accelerometer. J Athl Train 42:5-10, 2007

25. Hodgson VR: National Operating Committee on Standards for Athletic Equipment football helmet certification program. Med Sci Sports 7:225-232, 1975

26. Hodgson VR, Thomas LM: Comparison of head acceleration injury indices in cadaver skull fracture. SAE International Website. (http://papers.sae.org/710854) [Accessed September 27, 2011]

27. Hodgson VR, Thomas LM: Effect of long-duration impact on head. SAE International Website. (http://papers.sae.org/ 720956) [Accessed September 27, 2011]

28. Langlois JA, Rutland-Brown W, Wald MM: The epidemiology and impact of traumatic brain injury: a brief overview. J Head Trauma Rehabil 21:375-378, 2006

29. Maxwell WL, Povlishock JT, Graham DL: A mechanistic analysis of nondisruptive axonal injury: a review. J Neurotrauma 14:419-440, 1997

30. McCrea M, Hammeke T, Olsen G, Leo P, Guskiewicz K: Unreported concussion in high school football players: implications for prevention. Clin J Sport Med 14:13-17, 2004

31. McCrory P, Meeuwisse W, Johnston K, Dvorak J, Aubry M, Molloy M, et al: Consensus statement on concussion in sport-the 3rd International Conference on concussion in sport, held in Zurich, November 2008. J Clin Neurosci 16: 755-763, 2009

32. McElhaney JH: In search of head injury criteria. Stapp Car Crash J 49:v-xvi, 2005

33. McKee AC, Cantu RC, Nowinski CJ, Hedley-Whyte ET, Gavett BE, Budson AE, et al: Chronic traumatic encephalopathy in athletes: progressive tauopathy after repetitive head injury. J Neuropathol Exp Neurol 68:709-735, 2009

34. Mertz H, Prasad P, Irwin A: Injury risk curves for children and adults in frontal and rear collisions. SAE International Website. (http://papers.sae.org/973318) [Accessed September 27, 2011]

35. Mihalik JP, Bell DR, Marshall SW, Guskiewicz KM: Measurement of head impacts in collegiate football players: an investigation of positional and event-type differences. Neurosurgery 61:1229-1235, 2007

36. Mueller FO: Fatalities from head and cervical spine injuries occurring in tackle football: 50 years' experience. Clin Sports Med 17:169-182, 1998

37. National Center for Injury Prevention and Control: Report to Congress on Mild Traumatic Brain Injury in the United States: Steps to Prevent a Serious Public Health Problem. Atlanta: Centers for Disease Control and Injury Prevention, 2003

38. National Operating Committee on Standards for Athletic Equipment: Standard performance specification for newly manufactured football helmets. NOCSAE DOC (ND) $002-$ 98M09, 1-6, 2009. (http://www.nocsae.org/standards/pdfs/ Standards\%20'09/ND002-98m09\%20-\%20Mfr'd\%20FB\%20 Helmets\%20Standard\%20Performance.pdf) [Accessed September 18, 2011]

39. National Operating Committee on Standards for Athletic Equipment: Standard test method and equipment used in evaluating the performance characteristics of protective headgear/equipment. NOCSAE DOC (ND) 001-08M08B, 1-26. 2008, Overland Park, KS (http://www.nocsae.org/ standards/pdfs/Standards\%20'10/ND001-08m10-Drop\%20 Impact\%20Test\%20Method\%20.pdf ) [Accessed September $18,2011]$

40. Ommaya AK, Hirsch AE, Flamm ES, Mahone RH: Cerebral concussion in the monkey: an experimental model. Science 153:211-212, 1966

41. Pellman EJ, Viano DC, Tucker AM, Casson IR, Waeckerle JF: Concussion in professional football: reconstruction of game impacts and injuries. Neurosurgery 53:799-814, 2003

42. Pellman EJ, Viano DC, Withnall C, Shewchenko N, Bir CA, Halstead PD: Concussion in professional football: helmet testing to assess impact performance-part 11. Neurosurgery 58:78-96, 2006

43. Plassman BL, Havlik RJ, Steffens DC, Helms MJ, Newman TN, Drosdick D, et al: Documented head injury in early adulthood and risk of Alzheimer's disease and other dementias. Neurology 55:1158-1166, 2000

44. Prasad P, Mertz H: The position of the United States delegation to the ISO Working Group 6 on the use of HIC in the automotive environment. SAE International Website (http:// papers.sae.org/851246/) [Accessed September 27, 2011]

45. Rabadi MH, Jordan BD: The cumulative effect of repetitive concussion in sports. Clin J Sport Med 11:194-198, 2001

46. Rowson S, Beckwith JG, Chu JJ, Leonard DS, Greenwald RM, Duma SM: A six degree of freedom head acceleration measurement device for use in football. J Appl Biomech 27:8-14, 2011

47. Rowson S, Brolinson G, Goforth M, Dietter D, Duma S: Linear and angular head acceleration measurements in collegiate football. J Biomech Eng 131:061016, 2009

48. Schulz MR, Marshall SW, Mueller FO, Yang J, Weaver NL, Kalsbeek WD, et al: Incidence and risk factors for concussion in high school athletes, North Carolina, 1996-1999. Am J Epidemiol 160:937-944, 2004

49. Schwartz A: N.F.L. issues new guidelines on concussions. New York Times. December 2, 2009

50. Society of Automotive Engineers: Instrumentation for Impact Test-Part 1-Electronic Instrumentation. SAE International Website (http://standards.sae.org/j211/1_200707) [Accessed September 27, 2011]

51. Society of Automotive Engineers: Sign convention for vehicle crash testing. SAE International Website. (http://standards. sae.org/j1733_199412/) [Accessed September 27, 2011]

52. Takhounts EG, Eppinger RH, Campbell JQ, Tannous RE, Power ED, Shook LS: On the development of the SIMon Finite Element Head Model. Stapp Car Crash J 47:107-133, 2003

53. Thurman DJ, Branche CM, Sniezek JE: The epidemiology of sports-related traumatic brain injuries in the United States: recent developments. J Head Trauma Rehabil 13:1-8, 1998

54. Versace J: A review of the severity index. SAE International Website (http://papers.sae.org/710881) [Accessed September 27, 2011]

55. Viano DC, Pellman EJ, Withnall C, Shewchenko N: Concussion in professional football: performance of newer helmets in reconstructed game impacts-Part 13 . Neurosurgery 59: 591-606, 2006

56. Washington State Legislature: Zackary Lystedt Law, HB 1824 61st Legislature, 2009 Regular Session (2009) (http://apps.leg. wa.gov/documents/billdocs/2009-10/Pdf/Bills/Session\%20 Law\%202009/1824.SL.pdf) [Accessed September 28, 2011]

\section{Manuscript submitted June 21, 2011.}

Accepted September 6, 2011.

Please include this information when citing this paper: published online November 4, 2011; DOI: 10.3171/2011.9.JNS111059.

Address correspondence to: Adam Bartsch, Ph.D., Cleveland Clinic Spine Research Laboratory, Luth2-C, 1730 West 25th Street, Cleveland, Ohio 44113. email: bartsca@ccf.org. 